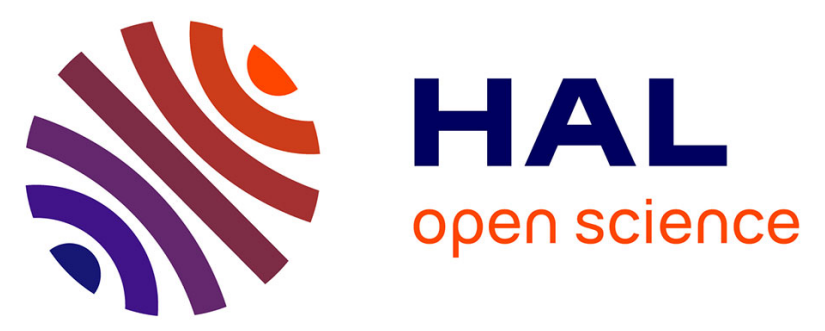

\title{
Anisotropic electrical, magnetic and thermal transport properties of the Al80Cr15Fe5 decagonal approximant
}

Janez Dolinsek, Stanislav Vrtnik, Ana Smontara, Marko Jagodic, Zvonko

Jaglicic, Birgitta Bauer, Peter Gille

\section{- To cite this version:}

Janez Dolinsek, Stanislav Vrtnik, Ana Smontara, Marko Jagodic, Zvonko Jaglicic, et al.. Anisotropic electrical, magnetic and thermal transport properties of the A180Cr $15 \mathrm{Fe} 5$ decagonal approximant. Philosophical Magazine, 2008, 88 (13-15), pp.2145-2153. 10.1080/14786430801946658. hal00513869

\section{HAL Id: hal-00513869 \\ https://hal.science/hal-00513869}

Submitted on 1 Sep 2010

HAL is a multi-disciplinary open access archive for the deposit and dissemination of scientific research documents, whether they are published or not. The documents may come from teaching and research institutions in France or abroad, or from public or private research centers.
L'archive ouverte pluridisciplinaire HAL, est destinée au dépôt et à la diffusion de documents scientifiques de niveau recherche, publiés ou non, émanant des établissements d'enseignement et de recherche français ou étrangers, des laboratoires publics ou privés. 


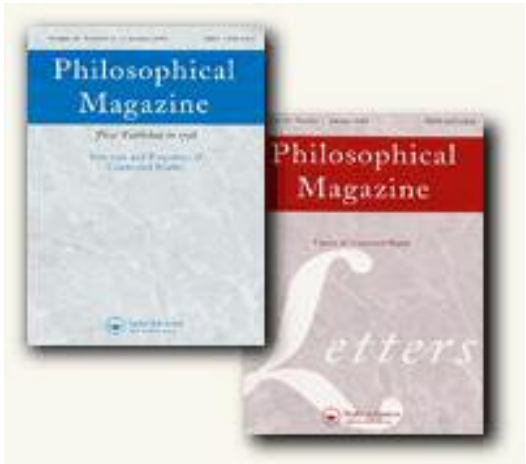

Anisotropic electrical, magnetic and thermal transport properties of the Al80Cr15Fe5 decagonal approximant

\begin{tabular}{|c|c|}
\hline Journal: & Philosophical Magazine \& Philosophical Magazine Letters \\
\hline Manuscript ID: & TPHM-07-Sep-0256.R2 \\
\hline Journal Selection: & Philosophical Magazine \\
\hline $\begin{array}{r}\text { Date Submitted by the } \\
\text { Author: }\end{array}$ & 15-Jan-2008 \\
\hline Complete List of Authors: & $\begin{array}{l}\text { Dolinsek, Janez; J. Stefan Institute, F5 } \\
\text { Vrtnik, Stanislav; J. Stefan Institute } \\
\text { Smontara, Ana; Institute of Physics } \\
\text { Jagodic, Marko; IMFM } \\
\text { Jaglicic, Zvonko; IMFM } \\
\text { Bauer, Birgitta; Uni-Muenchen } \\
\text { Gille, Peter; Uni-Muenchen }\end{array}$ \\
\hline Keywords: & $\begin{array}{l}\text { aluminium alloys, anisotropic properties, approximants, } \\
\text { quasicrystals }\end{array}$ \\
\hline Keywords (user supplied): & quasicrystalline approximants, physical properties, Al-Cr-Fe system \\
\hline
\end{tabular}

\section{๑) ScholarONE" \\ Manuscript Central}




\title{
Anisotropic electrical, magnetic and thermal transport properties of the
}

\section{$\mathrm{Al}_{80} \mathrm{Cr}_{15} \mathrm{Fe}_{5}$ decagonal approximant}

\author{
J. Dolinšek, ${ }^{1}$ S. Vrtnik, ${ }^{1}$ A. Smontara, ${ }^{2}$ M. Jagodič ${ }^{3}$ Z. Jagličić, ${ }^{3}$ B. Bauer, ${ }^{4}$ P. Gille ${ }^{4}$ \\ ${ }^{1}$ J. Stefan Institute, University of Ljubljana, Jamova 39, SI-1000 Ljubljana, Slovenia \\ ${ }^{2}$ Institute of Physics, Bijenička 46, POB 304, HR-10001 Zagreb, Croatia \\ ${ }^{3}$ Institute of Mathematics, Physics and Mechanics, Jadranska 19, Ljubljana, Slovenia \\ ${ }^{4}$ Ludwig-Maximilians-Universität München, Department of Earth and Environmental \\ Sciences, Crystallography Section, Theresienstrasse 41, D-80333 München, Germany
}

We present a study of the anisotropic magnetic, electrical and thermal transport properties of a single-crystalline $\mathrm{Al}_{80} \mathrm{Cr}_{15} \mathrm{Fe}_{5}$ approximant to the decagonal quasicrystal. Temperature-dependent electrical resistivity along the $b$ and $c$ crystalline directions shows nonmetallic behavior with a broad maximum, whereas it shows metallic positive temperature coefficient along the $a$ direction perpendicular to the $(b, c)$ atomic planes. The resistivity can be treated with the model of slow charge carriers, where the increased electron-phonon scattering upon raising the temperature induces transition from dominant Boltzmann (metallic) to dominant non-Boltzmann (insulatinglike) regime. Magnetic susceptibility and thermal conductivity show small anisotropy as well.

Short title: Anisotropic properties of $\mathrm{Al}_{80} \mathrm{Cr}_{15} \mathrm{Fe}_{5}$

Keywords: Quasicrystalline approximants, Physical properties, Al-Cr-Fe system Corresponding author: J. Dolinšek, phone: +386 14773 740, fax: +386 14773 191, email: jani.dolinsek@ijs.si 


\section{INTRODUCTION}

Decagonal $(d)$ phases are of special importance for the study of physical properties of quasicrystals (QCs). Their structure can be viewed as a periodic stack of quasiperiodic planes, so that $d$-QCs are two-dimensional QCs, whereas they are periodic crystals in a direction perpendicular to the quasiperiodic planes. The resistivity in the quasiperiodic (Q) and periodic $(\mathrm{P})$ directions can be consequently investigated on the same sample. Literature reports reveal that $d$-QCs exhibit anisotropy in their electronic and thermal transport properties (electrical resistivity $\rho$ [1-3], thermoelectric power $S$ [4], Hall coefficient $R_{H}[5,6]$, thermal conductivity $\kappa[7,8]$ and optical conductivity $\left.\sigma(\omega)[9]\right)$, when measured along the $\mathrm{Q}$ and $\mathrm{P}$ directions. The degree of anisotropy is related to the structural details of a particular decagonal phase, depending on the number of quasiperiodic layers in one periodic unit $[10,11]$. The most anisotropic case are the phases with just two layers, realized in $d$-Al-Cu-Co and $d$-Al-Ni-Co, where the periodicity length along the periodic axis is about $0.4 \mathrm{~nm}$ and the resistivity ratio at room temperature (RT) amounts typically $\rho_{Q} / \rho_{P} \approx 6-10$ [1-3]. Other $d$ phases contain more quasiperiodic layers in a periodic unit and show smaller anisotropies. In $d$ $\mathrm{Al}-\mathrm{Co}, d-\mathrm{Al}-\mathrm{Ni}$ and $d-\mathrm{Al}-\mathrm{Si}-\mathrm{Cu}-\mathrm{Co}$ there are four quasiperiodic layers with periodicity about $0.8 \mathrm{~nm}$ and the RT anisotropy is $\rho_{Q} / \rho_{P} \approx 2-4$ [4]. $d$-Al-Mn, $d$-Al-Cr and $d$ Al-Pd-Mn phases contain six layers with periodicity of about $1.2 \mathrm{~nm}$ and the anisotropy amounts $\rho_{Q} / \rho_{P} \approx 1.2-1.4$, whereas $d$-Al-Pd and $d$-Al-Cu-Fe phases with eight layers in a periodicity length of $1.6 \mathrm{~nm}$ are close to isotropic. A common property of $d$ phases is their metallic resistivity in the periodic direction, $\rho_{P}$, showing positive temperature coefficient (PTC) at metallic values (e.g. $\rho_{P}^{300 K} \approx 40 \mu \Omega c m$ in $d$-Al-Cu- 
Co and $d$-Al-Ni-Co [2]), whereas the considerably larger resistivity $\rho_{Q}$ in the quasiperiodic plane (e.g. $\rho_{Q}^{300 K} \approx 330 \mu \Omega \mathrm{cm}$ [2]) exhibits a NTC and usually also a maximum somewhere below RT or a leveling-off upon $T \rightarrow 0$. Anisotropies in the electronic and thermal transport properties similar to those in $d$-QCs were also observed in periodic structurally complex phases $\mathrm{Al}_{13} \mathrm{Co}(\mathrm{Fe})_{4}$ and the Taylor-phase $\mathrm{Al}_{3} \mathrm{Mn}$ [12]. In this paper we present a study of the anisotropic magnetic, electrical and thermal transport properties (magnetic susceptibility, electrical resistivity and thermal conductivity) along the three crystalline directions of a single-crystalline $\mathrm{Al}_{4}(\mathrm{Cr}, \mathrm{Fe})$ complex metallic alloy that is an excellent approximant to the decagonal phase with six atomic layers in a periodic unit. Electrical and thermal transport of this compound was already discussed in a recent paper [13].

\section{SAMPLE PREPARATION}

The investigated $\mathrm{Al}_{4}(\mathrm{Cr}, \mathrm{Fe})$ compound belongs to the class of body-centered orthorhombic phases $\mathrm{Al}_{4} \mathrm{TM}$ (transition metal) with $a \approx 1.25 \mathrm{~nm}, b \approx 1.25 \mathrm{~nm}$ and $c \approx 3.05 \mathrm{~nm}$, which coexist with the $d$-QC having a period of $1.25 \mathrm{~nm}$ along its periodic tenfold direction [14] (corresponding to the $a$ axis of $\mathrm{Al}_{4} \mathrm{TM}$ ). The $\mathrm{Al}_{4} \mathrm{TM}$ structure can be described as a periodic repetition of a sequence P'FPp'fp of six atomic layers stacked within one periodicity length of $1.25 \mathrm{~nm}$ along $a$, showing close structural relationship to the six-layer Al-TM $d$-QCs with the same periodicity. The block P'FP is composed of a flat layer $\mathrm{F}$ at $x=0$ and a puckered layer $\mathrm{P}$ at $x \approx a / 6$, 
whereas the puckered layer $\mathrm{P}^{\prime}$ is in mirror-reflecting position across the $\mathrm{F}$ layer. The block p'fp equals the block P'FP translated by $(a / 2, b / 2, c / 2)$.

The single crystal used in our study was grown from an incongruent Al-rich melt of initial composition $\mathrm{Al}_{87} \mathrm{Cr}_{7} \mathrm{Fe}_{6}$ by the Czochralski method using a native seed. The composition of the sample (rounded to the closest integers) was $\mathrm{Al}_{80} \mathrm{Cr}_{15} \mathrm{Fe}_{5}$ and its structure could be assigned to the orthorhombic phase, previously described by Deng et al. [14], with the following crystallographic parameters: Pearson's symbol ol366-59.56, space group Immm (No. 71), unit cell parameters $a=1.2500(6) \mathrm{nm}$, $b=1.2617(2) \mathrm{nm}, c=3.0651(8) \mathrm{nm}$ and 306.44 atoms in the giant unit cell. In order to perform crystalline-direction-dependent studies, we prepared three bar-shaped samples of dimensions $2 \times 2 \times 8 \mathrm{~mm}^{3}$ with their long axes along the three crystallographic directions of the orthorhombic unit cell. The $\left[\begin{array}{lll}1 & 0 & 0\end{array}\right]$ direction (designated in the following as $a$ ) corresponds to the periodic direction in $d$-QCs, whereas the $\left[\begin{array}{lll}0 & 1 & 0\end{array}\right](b)$ and $\left[\begin{array}{lll}0 & 0 & 1\end{array}\right](c)$ directions lie within the atomic planes (corresponding to the quasiperiodic directions in $d$-QCs).

\section{MAGNETIC SUSCEPTIBILITY}

The magnetization of the $\mathrm{Al}_{80} \mathrm{Cr}_{15} \mathrm{Fe}_{5}$ as a function of the magnetic field, $M(H)$, and the temperature-dependent magnetic susceptibility, $\chi(T)$, were measured with a Quantum Design SQUID magnetometer, equipped with a $50 \mathrm{kOe}$ magnet. The susceptibility $\chi=M / H$ was investigated in the temperature interval between 300 and $2 \mathrm{~K}$ in a magnetic field $H=1 \mathrm{kOe}$ (Fig. 1a), which was directed along each of the three 
crystalline directions. The respective susceptibilities are labeled as $\chi_{a}, \chi_{b}$ and $\chi_{c}$. All three susceptibilities exhibit a simple paramagnetic behavior and were analyzed with the Curie-Weiss law

$$
\chi_{j}=\chi_{0 j}+\frac{C_{j}}{T-\theta_{j}}
$$

where the subscript $j$ denotes the crystalline direction, $\chi_{0 j}$ is a temperatureindependent term, $C_{j}$ the Curie constant and $\theta_{j}$ the Curie-Weiss temperature. The fits are shown as solid lines in Fig. 1a and the fit parameter values are given in Table 1. We observe that all three susceptibilities are very similar in magnitude and temperaturedependence. A weak anisotropy $\chi_{c}>\chi_{b}>\chi_{a}$ can be noticed.

The $M(H)$ dependence up to $50 \mathrm{kOe}$ along the three crystalline directions is displayed in Fig. 1b. We observe that there is almost no anisotropy for the two in-plane crystalline directions $b$ and $c$, whereas the magnetization along $a$ is slightly smaller. Further $M(H)$ analysis is hindered by the fact that there exist two different kinds of magnetic moments $(\mathrm{Cr}$ and $\mathrm{Fe})$ in the samples, so that one should use a sum of two Brillouin functions to describe the paramagnetic magetization. Since too many parameters are then involved (that can compensate each other in the fit procedure), we skip further analysis.

\section{ELECTRICAL AND THERMAL TRANSPORT PROPERTIES}

\section{a) Electrical resistivity}

Electrical resistivity was measured between 300 and $2 \mathrm{~K}$ using the standard fourterminal technique and the data are displayed in Fig. 2. The resistivity is the lowest 
along the $a$ axis perpendicular to the atomic planes. $\rho_{a}$ shows a PTC in the whole investigated temperature interval and a RT value $\rho_{a}^{300 K}=297 \mu \Omega \mathrm{cm}$. The resistivities within the atomic planes are higher and exhibit different temperaturedependencies with a broad maximum, where the temperature coefficient is reversed. $\rho_{b}$ exhibits a maximum at about $125 \mathrm{~K}$ with the peak value $375 \mu \Omega \mathrm{cm}$ and the RT value $\rho_{b}^{300 K}=371 \mu \Omega \mathrm{cm}$. The resistivity $\rho_{c}$ is the highest; its maximum value $413 \mu \Omega \mathrm{cm}$ occurs at $100 \mathrm{~K}$ and the RT value is $\rho_{c}^{300 \mathrm{~K}}=407 \mu \Omega \mathrm{cm}$. At RT, the ratios of the resistivities amount $\rho_{c} / \rho_{a}=1.37, \rho_{b} / \rho_{a}=1.25$ and $\rho_{c} / \rho_{b}=1.10$. The resistivity of $\mathrm{Al}_{80} \mathrm{Cr}_{15} \mathrm{Fe}_{5}$ is thus analogous to $d$-QCs [2-4], where the resistivity perpendicular to the quasiperiodic atomic planes also shows metallic PTC and its value is smaller than the in-plane resistivity, which exhibits a nonmetallic character with NTC on the high-temperature side of the maximum in $\rho(T)$ and PTC below.

In an anisotropic crystal, the electrical conductivity $\boldsymbol{\sigma}$ is generally a symmetric (and diagonalizable) tensor, relating the current density $\vec{j}$ to the electric field $\vec{E}$ via the relation $j_{i}=\sum_{j} \sigma_{i j} E_{j}$, where $i, j=x, y, z$ denote crystalline directions. The tensorial ellipsoid exhibits the same symmetry axes as the crystallographic structure. For our orthorhombic $\mathrm{Al}_{80} \mathrm{Cr}_{15} \mathrm{Fe}_{5}$ crystal this implies that the conductivity tensor is diagonal in the basis of the crystalline directions $a, b$ and $c$. The geometry of our samples (their long axes were along the three crystalline directions) and the direction of the electric field applied along their long axes imply that diagonal elements $\sigma_{x x}=\sigma_{a}$, $\sigma_{y y}=\sigma_{b}$ and $\sigma_{z z}=\sigma_{c}$ were measured in our experiments. The temperature 
dependence of each of these elements was then analyzed using the theory of slow charge carriers by Trambly de Laissardière et al. [15], which applies to any diagonal element of the conductivity tensor (in [15], $\sigma_{x x}$ is considered, but $x$ may be any crystalline direction).

Trambly de Laissardière et al. have shown [15] that the semiclassical (BlochBoltzmann) model of conduction breaks down when the mean free path of charge carriers is smaller than a typical extension of their wave function. This situation is realized for sufficiently slow charge carriers (where low electronic velocity is a consequence of weak dispersion of the electronic bands) and leads to a transition from a metallic to an insulatinglike regime when scattering by defects or temperature effects increases. According to the Einstein relation, the conductivity $\sigma$ depends on the electronic density of states (DOS) $g(E)$ and the spectral diffusivity $D(E)$ within the thermal interval of a few $k_{B} T$ around the Fermi level $E_{F}$. In the case of slowly varying metallic DOS around $E_{F}$ it is permissible to replace $g(E)$ by $g\left(E_{F}\right)$. For the diffusion constant it was shown [15] that it can be written as $D=v^{2} \tau+L^{2}(\tau) / \tau$, where $v$ is the electronic velocity, $\tau$ the scattering time and $L^{2}(\tau)$ is the non-ballistic (non-Boltzmann) contribution to the square of spreading of the quantum state at energy $E$ due to diffusion, averaged on a time scale $\tau . L(\tau)$ is bounded by the unit cell length and saturates to a constant value already for short averaging time. The dc conductivity of the system in the crystalline direction $j$ can be written as

$$
\sigma_{j}=\sigma_{B j}+\sigma_{N B j}=e^{2} g\left(E_{F}\right) v_{j}^{2} \tau_{j}+e^{2} g\left(E_{F}\right) \frac{L_{j}^{2}\left(\tau_{j}\right)}{\tau_{j}},
$$


where $\sigma_{B j}$ is the Boltzmann contribution and $\sigma_{N B j}$ is the non-Boltzmann contribution.

The scattering rate $\tau^{-1}$ will generally be a sum of a temperature- and orientationindependent rate $\tau_{0}^{-1}$ due to scattering by quenched defects and a temperaturedependent term due to scattering by phonons $\tau_{p}^{-1}$. The anisotropy of the atomic structure implies that the phonon spectrum will also be anisotropic, so that the scattering rate will generally depend on the crystalline direction, $\tau_{j}^{-1}=\tau_{0}^{-1}+\tau_{p j}^{-1}$. In the simplest case, $\tau_{p j}$ can be phenomenologically written as a power-law of temperature, $\tau_{p j}=\beta_{j} / T^{\alpha_{j}}$. Assuming that $L_{j}^{2}\left(\tau_{j}\right)$ can be replaced by its limiting value, a constant $L_{j}^{2}$, Eq. (2) yields a minimum in the conductivity $\sigma_{j}$ as a function of $\tau_{j}$ or temperature (or equivalently, there is a maximum in the resistivity $\rho_{j}=\sigma_{j}^{-1}$ ) at the condition $\tau_{j}=L_{j} / v_{j}$. Above the resistivity maximum, the non-Boltzmann contribution prevails and the resistivity exhibits a nonmetallic NTC, whereas below the maximum, the resistivity exhibits a metallic PTC due to dominant Boltzmann contribution. The resistivity maxima, as observed for $\rho_{b}$ and $\rho_{c}$ in Fig. 2, can thus be considered as a consequence of a crossover from dominant ballistic conductivity at low$\mathrm{T}$ to dominant non-ballistic conductivity at high-T due to small velocities of the charge carriers.

Defining $A_{j}=e^{2} g\left(E_{F}\right) v_{j}^{2} \tau_{0}, B_{j}=e^{2} g\left(E_{F}\right) L_{j}^{2} / \tau_{0}$ and $C_{j}=\tau_{0} / \beta_{j}$, Eq.

(2) can be rewritten as 


$$
\sigma_{j}=\frac{A_{j}}{1+C_{j} T^{\alpha_{j}}}+B_{j}\left(1+C_{j} T^{\alpha_{j}}\right)
$$

that contains four crystalline-direction-dependent fit parameters $A_{j}, B_{j}, C_{j}$ and $\alpha_{j}$ (the last two always appear in a product $C_{j} T^{\alpha_{j}}$ ). The zero-temperature conductivity is obtained as $\sigma_{j}^{0}=A_{j}+B_{j}$. In the regime of dominant scattering by quenched defects, $\tau_{0} / \tau_{p j}=C_{j} T^{\alpha}<<1$, normally realized at low temperatures, expansion of Eq. (3) yields the low-temperature form of the conductivity $\sigma_{j}=\sigma_{j}^{0}-\sigma_{1} T^{\alpha_{j}}$ (provided $A_{j}>B_{j}$ ). This can be viewed as a generalized Bloch-Grüneisen law that yields a metallic PTC resistivity. In the other extreme of dominant phonon scattering, $\tau_{0} / \tau_{p j}=C_{j} T^{\alpha_{j}}>>1$, normally realized at high temperatures, Eq. (3) yields the high-temperature form of the conductivity as $\sigma_{j}=\sigma_{2} T^{\alpha_{j}}$, yielding an insulator-like NTC resistivity. The relative magnitudes of $A_{j}, B_{j}$ and $C_{j} T^{\alpha_{j}}$ coefficients thus determine the temperature-dependence of the resistivity within a given temperature range, which can either be in the metallic or insulating-like regimes, or at a crossover between these two regimes (in which case the resistivity exhibits a maximum). Since these coefficients depend on the electronic structure of the investigated compound $\left(g\left(E_{F}\right)\right.$ and $\left.v_{j}\right)$, its crystallographic details $\left(L_{j}\right)$, defect concentration $\left(\tau_{0}\right)$ and phononic spectrum $\left(\tau_{p j}\right)$, they are specific to a given structure and sample purity.

The fits of the resistivities with Eq. (3) are displayed in Fig. 2 as solid lines and the fit parameters are collected in Table 2. The fits are excellent for all three crystalline 
directions. The $A_{j}$ parameter values enable us to estimate the anisotropy of the electronic average velocities along the three crystalline directions. We get $v_{a} / v_{b}=\sqrt{A_{a} / A_{b}}=1.13, v_{a} / v_{c}=1.17$ and $v_{b} / v_{c}=1.04$, so that the velocity is the highest along the $a$ axis perpendicular to the atomic planes and there is also small anisotropy within the atomic planes. The parameters $C_{j}$ and $\alpha_{j}$ describe the anisotropy of the electron-phonon scattering rate. Anisotropy of the phononic spectrum of $\mathrm{Al}_{80} \mathrm{Cr}_{15} \mathrm{Fe}_{5}$ is reflected in the anisotropy of the lattice thermal conductivity $\kappa_{p h}$, to be presented next.

Here it should be mentioned that a maximum in the resistivity at low temperatures is also predicted by the theory of weak localization [16], frequently used to analyze the temperature-dependent resistivity of icosahedral QCs. Weak localization is considered to introduce small temperature-dependent correction to the Boltzmann conductivity due to spin-orbit and inelastic scattering processes of electrons. However, while the validity of the weak localization concept is restricted to low temperatures, the theory of slow charge carriers of Eq. (3) is applicable at all temperatures and does not involve any electron localization.

\section{b) Thermal conductivity}

The thermal conductivity $\kappa$ of $\mathrm{Al}_{80} \mathrm{Cr}_{15} \mathrm{Fe}_{5}$ was measured along the three crystallographic directions using an absolute steady-state heat-flow method. The thermal flux through the samples was generated by a $1 \mathrm{k} \Omega \mathrm{RuO}_{2}$ chip-resistor, glued to one end of the sample, while the other end was attached to a copper heat sink. The temperature gradient across the sample was monitored by a chromel-constantan 
differential thermocouple. The phononic contribution $\kappa_{p h}=\kappa-\kappa_{e l}$ was extracted by subtracting the electronic contribution $\kappa_{e l}$ from the total conductivity using the Wiedemann-Franz law and the measured electrical resistivity data. Phononic thermal conductivity is displayed in Fig. 3, where we observe that the conductivity $\kappa_{p h}^{a}$ along the $a$ direction in the low-T regime below $50 \mathrm{~K}$ (which can be associated with the regime where umklapp processes are still ineffective) is the highest, whereas the two inplane conductivities $\kappa_{p h}^{b}$ and $\kappa_{p h}^{c}$ are somewhat smaller and also show very weak inplane anisotropy. The observed anisotropy of $\kappa_{p h}$ thus gives qualitative support to the presumption that the phononic spectrum of $\mathrm{Al}_{80} \mathrm{Cr}_{15} \mathrm{Fe}_{5}$ is spatially anisotropic and justifies the use of a crystalline-direction-dependent phonon scattering rate $\tau_{p j}^{-1}$ in the analysis of the electrical resistivity.

\section{CONCLUSIONS}

The $\mathrm{Al}_{80} \mathrm{Cr}_{15} \mathrm{Fe}_{5}$ complex intermetallic compound is a periodic approximant to the decagonal phase and single-crystalline samples of high structural perfection could recently be grown. Due to the six-layer structure of the $\mathrm{Al}_{4} \mathrm{TM}$ phase, the anisotropy in the transport properties is smaller than in the two- and four-layer structures, but still pronounced. The velocity of the charge carriers is just in the range where temperature induces transition from dominant Boltzmann (metallic) to dominant non-Boltzmann (insulatinglike) regimes, as demonstrated in the appearance of the resistivity maximum for specific crystalline directions. Consequently, a temperature-dependent electronic diffusion coefficient pertinent to slow charge carriers enables unified treatment of both 
regimes. The anisotropy of the resistivity is related to the anisotropy of the electronic band structure that is responsible for the anisotropy of the electronic velocity along different crystalline directions. A preliminary discussion of the velocity anisotropy in $d$ QCs in relation to the number of quasiperiodic layers within one periodic unit is given elsewhere [11], where the two-layer case is shown to be the most anisotropic one. The above approach of slow charge carriers has been successfully applied to the electrical resistivity, whereas implementation of this concept to other transport coefficients still has to be performed.

Acknowledgements: This work was done within the activities of the $6^{\text {th }}$ Framework EU Network of Excellence "Complex Metallic Alloys" (Contract No. NMP3-CT-2005$500140)$. 
Table 1: Fit parameters of the Curie-Weiss paramagnetic susceptibility (solid curves in Fig. 1a, as calculated from Eq. (1)) with the magnetic field applied along the three crystalline directions $a, b$ and $c$.

\begin{tabular}{|c|c|c|c|}
\hline & $\chi_{o j}\left(\times 10^{-6}\right.$ emu $\left./ g\right)$ & $C_{j}\left(\times 10^{-5}\right.$ emuK $\left./ g\right)$ & $\theta_{j}(K)$ \\
\hline$a$ & 1.4 & 2.2 & -6.0 \\
\hline$b$ & 1.7 & 3.6 & -4.5 \\
\hline$c$ & 1.9 & 4.2 & -5.4 \\
\hline
\end{tabular}


Table 2: Fit parameters of the electrical resistivity (solid curves in Fig. 2, as calculated from Eq. (3)). The units of the coefficients $C_{j}$ are chosen so that the temperature in the expression $C_{j} T^{\alpha_{j}}$ is dimensionless.

\begin{tabular}{|c|c|c|c|}
\hline \multicolumn{4}{|c|}{ crystalline direction $a$} \\
\hline$A_{a}(\mu \Omega c m)^{-1}$ & $B_{a}(\mu \Omega c m)^{-1}$ & $C_{a}$ & $\alpha_{a}$ \\
\hline $2.16 \times 10^{-3}$ & $1.32 \times 10^{-3}$ & $4.40 \times 10^{-3}$ & 0.70 \\
\hline \multicolumn{4}{|c|}{ crystalline direction $b$} \\
\hline$A_{b}(\mu \Omega c m)^{-1}$ & $B_{b}(\mu \Omega c m)^{-1}$ & $C_{b}$ & $\alpha_{b}$ \\
\hline $1.70 \times 10^{-3}$ & $1.04 \times 10^{-3}$ & $1.27 \times 10^{-2}$ & 0.64 \\
\hline \multicolumn{4}{|c|}{ crystalline direction $c$} \\
\hline$A_{c}(\mu \Omega c m)^{-1}$ & $B_{c}(\mu \Omega c m)^{-1}$ & $C_{c}$ & $\alpha_{c}$ \\
\hline $1.57 \times 10^{-3}$ & $9.34 \times 10^{-4}$ & $2.06 \times 10^{-2}$ & 0.57 \\
\hline
\end{tabular}




\section{References:}

[1] T. Shibuya, T. Hashimoto, S. Takeuchi, J. Phys. Soc. Jpn. 59, 1917 (1990).

[2] S. Martin, A.F. Hebard, A.R. Kortan, F.A. Thiel, Phys. Rev. Lett. 67, 719 (1991).

[3] Wang Yun-ping, Zhang Dian-lin, Phys. Rev. B 49, 13204 (1994).

[4] Lin Shu-yuan, Wang Xue-mei, Lu Li, Zhang Dian-lin, L.X. He, K.X. Kuo, Phys. Rev. B 41, 9625 (1990).

[5] Zhang Dian-lin, Lu Li, Wang Xue-mei, Lin Shu-yuan, Phys. Rev. B 41, 8557 (1990).

[6] Wang Yun-ping, Zhang Dian-lin, L.F. Chen, Phys. Rev. B 48, 10542 (1993).

[7] Zhang Dian-lin, Cao Shao-chun, Wang Yun-ping, Lu Li, Wang Xue-mei, X.L. Ma, K.H. Kuo, Phys. Rev. Lett. 66, 2778 (1991).

[8] K. Edagawa, M.A. Chernikov, A.D. Bianchi, E. Felder, U. Gubler, H.R. Ott, Phys. Rev. Lett. 77, 1071 (1996).

[9] D.N. Basov, T. Timusk, F. Barakat, J. Greedan, B. Grushko, Phys. Rev. Lett. 72, 1937 (1994).

[10] M. Krajčí, J. Hafner, Phys. Rev. B 58, 5378 (1998).

[11] G. Trambly de Laissardière, T. Fujiwara, Phys. Rev. B 50, 9843 (1994).

[12] P. Volkov, S.J. Poon, Phys. Rev. B 52, 12685 (1995).

[13] J. Dolinšek, P. Jeglič, M. Komelj, S. Vrtnik, A. Smontara, I. Smiljanić, A. Bilušić, J. Ivkov, D. Stanić, E.S. Zijlstra, B. Bauer, P. Gille, Phys. Rev. B 76, 174207 (2007). [14] D.W. Deng, Z.M. Mo, K.H. Kuo, J. Phys.: Condens. Matter 16, 2283 (2004).

[15] G. Trambly de Laissardière, J.-P. Julien, D. Mayou, Phys. Rev. Lett. 97, 026601 (2006).

[16] H. Fukuyama, K. Hoshino, J. Phys. Soc. Jpn. 50, 2131 (1981). 


\section{Figure captions:}

Fig. 1: (Color online) (a) Temperature-dependent magnetic susceptibility $\chi$ in a field $H=1 \mathrm{kOe}$ oriented along the three crystalline directions $a, b$ and $c$ of the orthorhombic unit cell of $\mathrm{Al}_{80} \mathrm{Cr}_{15} \mathrm{Fe}_{5}$. Solid lines are Curie-Weiss fits with Eq. (1) and the fit parameters are given in Table 1. (b) Magnetization $M$ as a function of the magnetic field at $T=2 \mathrm{~K}$.

Fig. 2: (Color online) Temperature-dependent electrical resistivity of $\mathrm{Al}_{80} \mathrm{Cr}_{15} \mathrm{Fe}_{5}$ along the three crystalline directions $a, b$ and $c$ of the orthorhombic unit cell. Solid lines are fits with Eq. (3) and the fit parameter values are given in Table 2.

Fig. 3: (Color online) Lattice thermal conductivity $\kappa_{p h}$ of $\mathrm{Al}_{80} \mathrm{Cr}_{15} \mathrm{Fe}_{5}$ along the three crystalline directions $a, b$ and $c$ of the orthorhombic unit cell, obtained by subtracting the electronic contribution from the total thermal conductivity using the WiedemannFranz law. 

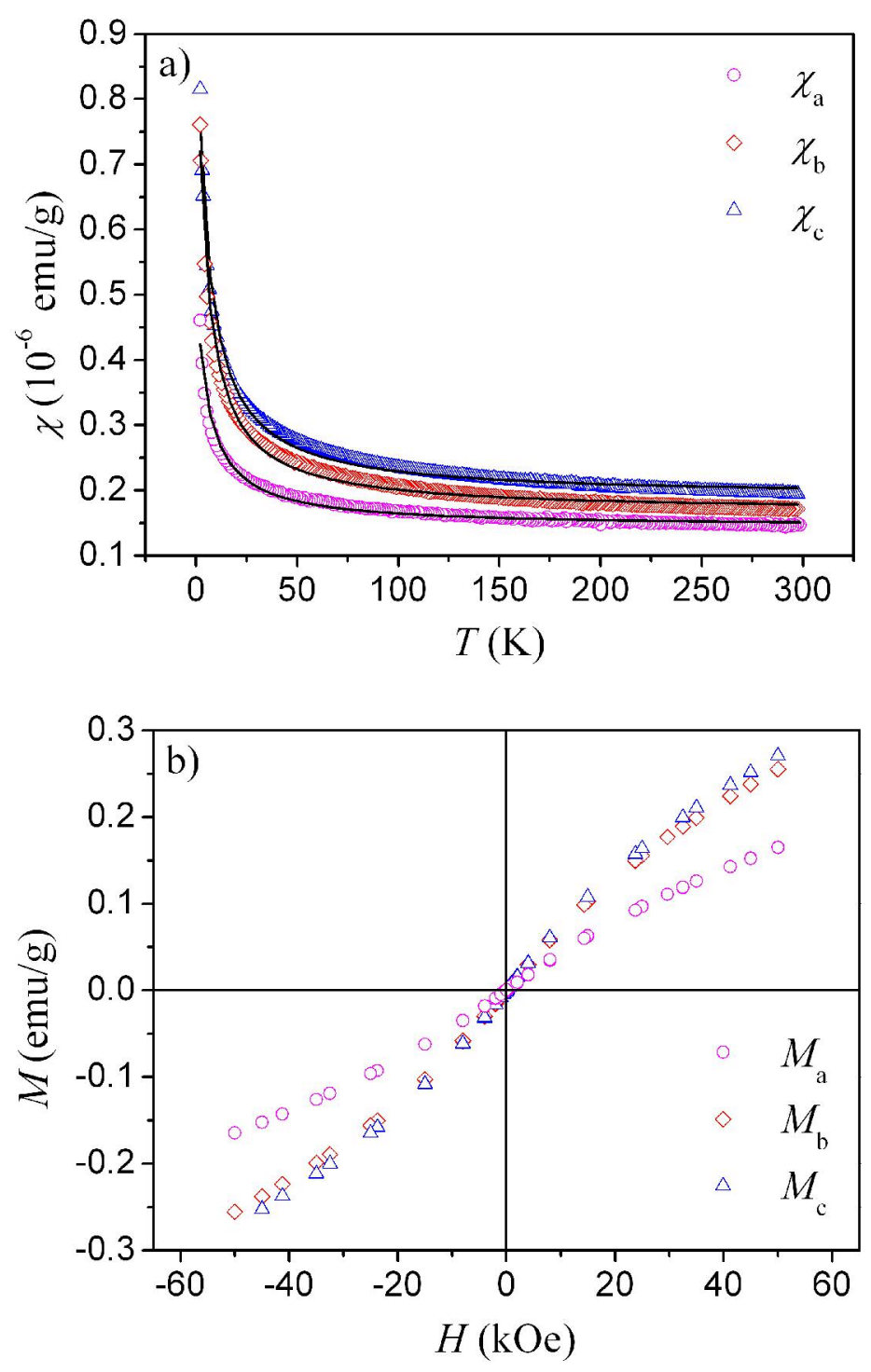

$250 \times 371 \mathrm{~mm}(300 \times 300$ DPI $)$ 


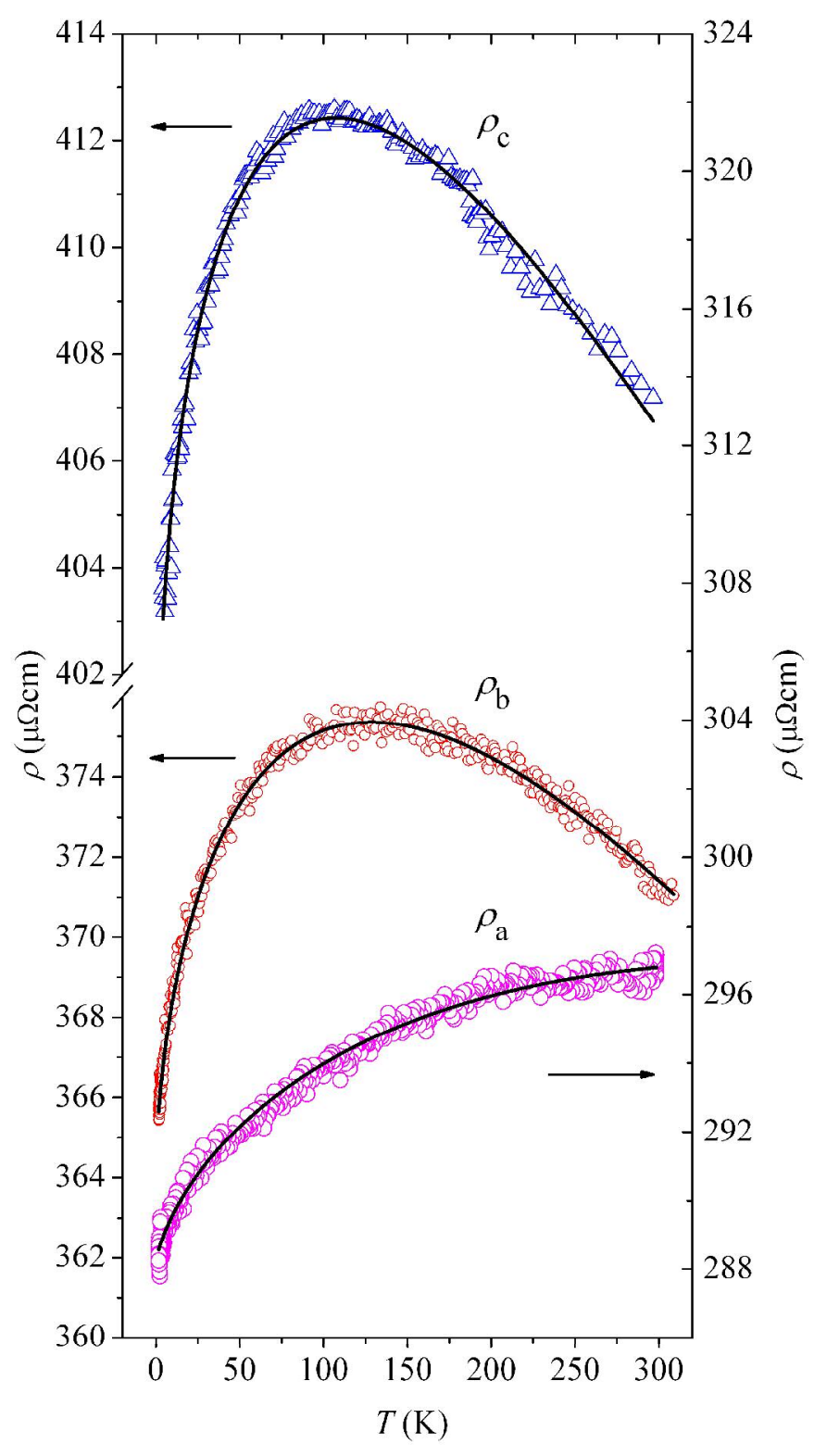

$996 \times 1794 \mathrm{~mm}(72 \times 72 \mathrm{DPI})$ 


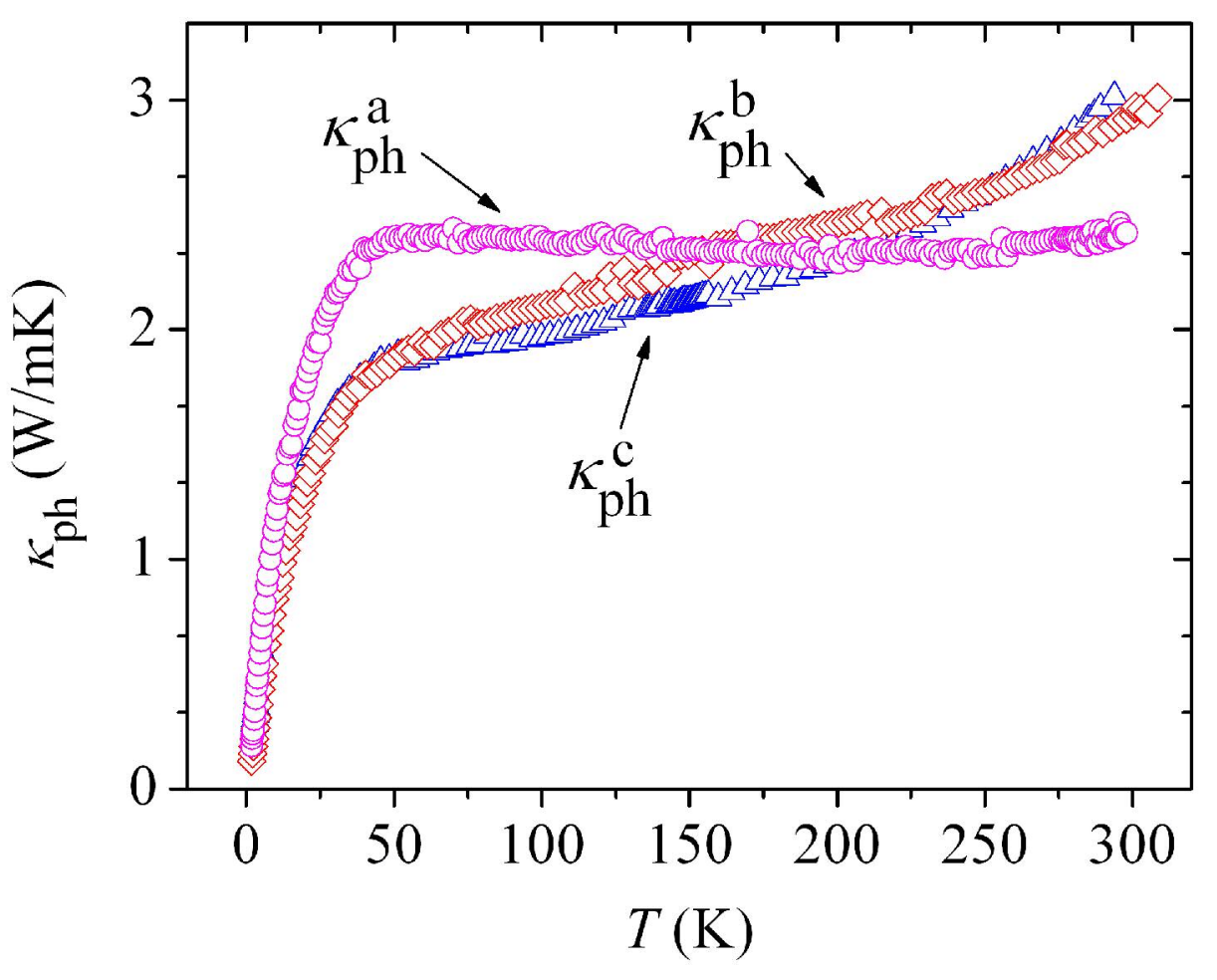

$916 \times 708 \mathrm{~mm}(72 \times 72$ DPI $)$ 